\title{
Initial 'TTP Map-Defect' of Computed Tomography Perfusion as a Predictor of Hemorrhagic Transformation of Acute Ischemic Stroke
}

\author{
Mizuya Shinoyama $^{a, c}$ Jyoji Nakagawara ${ }^{a}$ Hiroshi Yoneda $^{c}$

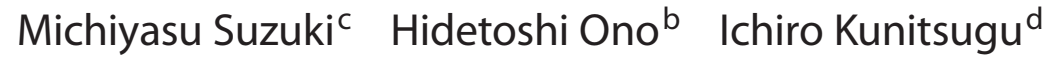 \\ Kenji Kamiyama $^{a}$ Toshiaki Osato $^{a}$ Hirohiko Nakamura $^{a}$ \\ Departments of a Neurosurgery and ${ }^{b}$ Radiology, Nakamura Memorial Hospital, Sapporo, and \\ Departments of ${ }^{c}$ Neurosurgery and ${ }^{d}$ Public Health, Yamaguchi University School of Medicine, \\ Yamaguchi, Japan
}

\section{Key Words}

Hemorrhagic transformation $\cdot$ Computed tomography perfusion $\cdot$ Time to peak $\cdot$ Ischemic stroke

\begin{abstract}
Background: Hemorrhagic transformation (HT) following acute ischemic stroke is a major problem, especially for the indication of reperfusion therapy including intravenous administration of recombinant tissue plasminogen activator (IV rt-PA). The specific predictive factors of HT have not yet been established. The present study evaluated the findings of computed tomography perfusion (CTP) images as predictors of subsequent $\mathrm{HT}$ to identify patients with low HT risk for reperfusion therapy such as IV rt-PA. Methods: We retrospectively reviewed 68 consecutive stroke patients (41 males; mean age 72.9 years) with steno-occlusive lesions in the major trunk, including 10 patients who underwent IV rt-PA. Each HT was detected on a follow-up T2*weighted magnetic resonance image until 2 weeks after stroke onset and categorized into four groups [hemorrhagic infarction (HI) type 1 and 2, and parenchymal hematoma (PH) type 1 and 2] according to the European Cooperative Acute Stroke Study (ECASS) classification. We assessed clinical features and radiological findings between the $\mathrm{HT}$ and non-HT groups or the $\mathrm{PH} 2$ and non-PH2 groups. The efficacy of initial time to peak (TTP) mapping of CTP for predicting HT or PH2 was evaluated. Results: Thirty-four patients (50\%) developed subsequent HT: 18 (52.9\%) had $\mathrm{HI}$ and 16 (47.1\%) had $\mathrm{PH}$, including $9 \mathrm{PH} 2$ patients (13.2\%). IV rt-PA was not signifi-
\end{abstract}


cantly associated with $\mathrm{HT}$ or $\mathrm{PH} 2$ occurrence. Forty of the 68 patients (59\%) revealed defect areas on the initial TTP mapping (TTP map-defect), and 34 of these 40 patients (85\%) developed secondary HT and 9 patients (22.5\%) developed PH2. Initial 'TTP map-defect' was significantly associated with the occurrence of HT $(p<0.0001)$ and PH2 $(p=0.0070)$. Thirty of the 34 patients $(88.2 \%)$ in the HT group experienced delayed recanalization of the occluded vessels, in contrast to only 8 of the 34 patients (23.6\%) in the non-HT group. All patients of the PH2 group showed recanalization ( $p=0.0042$ ). In 40 'TTP map-defect'-positive patients, delayed recanalization was associated with the occurrence of HT $(p<0.0001)$ and PH2 $(p=0.0491)$. All 28 patients without 'TTP map-defect' did not develop HT, including 8 patients (28.6\%) with delayed recanalization. Conclusions: Initial 'TTP map-defect' of CTP could accurately predict HT risk including PH2 risk and identify low-risk patients even in the delayed period.

Copyright $\odot 2013$ S. Karger AG, Basel

\section{Introduction}

Hemorrhagic transformation (HT) in acute ischemic stroke occurs either spontaneously [1] or after thrombolytic therapy [2-4]. Thrombolytic therapy consisting of intravenous administration of recombinant tissue plasminogen activator (IV rt-PA) reduces ischemic brain injury in some patients but causes symptomatic HT in $6-10 \%$ of treated patients, resulting in a mortality rate of $45-61 \%$ [2-4]. Therefore, thrombolytic therapy is considered to be a double-edged sword. Partly because of the risk of hemorrhagic complication, only $3.4-5.2 \%$ of all transferred ischemic stroke patients could undergo IV rt-PA in the USA in 2009 [5]. Therefore, pre-treatment selection to identify patients with low risk of HT could increase the opportunity for reperfusion therapy. The estimation of the individual HT risk is more important because of the widened therapeutic time window for IV rt-PA beyond $4.5 \mathrm{~h}[6]$.

Various clinical factors such as etiologies or underlying medical histories have been proposed as indicators for HT, but the specific predictors have not yet been established [7-12]. Initial radiological findings of extensive early ischemic lesions or poor collateral flow on conventional computed tomography (CT) or magnetic resonance image (MRI) could be predictors of HT; however, they have not yet been shown to predict accurate patients' risk of HT.

Recently, perfusion imaging has become important in the diagnosis of acute ischemic stroke. Bolus-chase CT perfusion (CTP) or MR perfusion (MRP) uses dynamic contrastenhanced data to produce parametric color overlay maps of cerebral blood flow (CBF), cerebral blood volume (CBV), mean transit time (MTT), and time to peak (TTP). These parameters have already been proved to accurately indicate focal brain perfusion and applied to clinical use in acute cerebral infarction [13-16]. Perfusion images have been reported to predict the patient's hemorrhagic outcome by assessing parameters such as permeability of contrast [17], CBV [18] or relative MTT [19]. However, their results are not suitable to anticipate the therapeutic time window of IV rt-PA.

Among the parameters, in particular, TTP mapping shows focal hypoperfusion at higher sensitivity [20-24]. Moreover, TTP mapping has advantages in short data processing time without complex calculations such as deconvolution, so it has been widely applied in the clinical setting. However, the predictive value of TTP mapping for estimating the risk of HT in patients with acute ischemic stroke has not been evaluated.

The present study evaluated the efficacy of initial TTP mapping on admission as a predictor of subsequent HT to identify patients with low HT risk [25] for reperfusion therapy. 


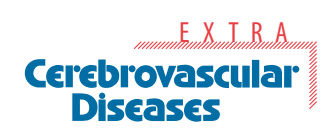

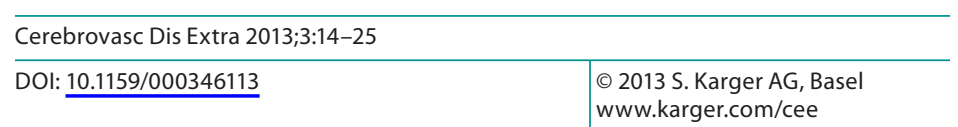

www.karger.com/cee

Shinoyama et al.: Initial 'TTP Map-Defect' of Computed Tomography Perfusion as a

Predictor of Hemorrhagic Transformation of Acute Ischemic Stroke

\section{Methods}

\section{Inclusion Criteria}

We retrospectively reviewed the radiological findings of all patients with ischemic stroke who were admitted to the stroke service of the Nakamura Memorial Hospital from January to September 2010. We evaluated patients with a steno-occlusive lesion in the intra- or extracranial major cerebral vessels who underwent both CTP and MRI as initial imaging assessments within the first $24 \mathrm{~h}$ of stroke onset.

The criteria for the study were as follows: initial CT and MRI showed no intracranial hemorrhage and a follow-up MRI was performed at least twice, the first on the day after stroke onset (day 2) and the second within 2 weeks. Both MRIs included diffusion-weighted imaging, T2*-weighted imaging, and magnetic resonance angiography (MRA).

rt-PA was administered according to the Japanese guidelines [26, 27]. Patients who died of extra-cranial causes within the first 2 weeks were excluded.

\section{Imaging Procedure}

All CTP studies were performed using a 128-slice CT scanner (SOMATOM Definition AS+; Siemens, Forchheim, Germany) with adaptive 4D-spiral mode. The technique is based on a constant periodic bidirectional table movement. The imaging parameters were $80 \mathrm{kV}$ and $180 \mathrm{mAs}, 20$ spiral images with an image volume of $96 \mathrm{~mm}$ on the $\mathrm{z}$-axis, and a travel time of $1.5 \mathrm{~s}$ per spiral. Reconstructed images of $5 \mathrm{~mm}$ were obtained for every $1 \mathrm{~mm}$ of the total imaging volume.

A bolus of $40 \mathrm{ml}$ of the non-ionic contrast material Iohexol 350 (Omnipaque 350; Daiichi-Sankyo, Tokyo, Japan) was injected through a 20 -gauge cannula placed in the cubital vein at a flow rate of $5 \mathrm{ml} / \mathrm{s}$ followed by $20 \mathrm{ml}$ of saline chaser.

MRI was performed with a 1.5-T clinical whole-body unit (Magnetom Avanto; Siemens, Erlangen, Germany). The parameters of T2*-weighted gradient-echo imaging were repetition time (TR) $650 \mathrm{~ms}$, echo time (TE) $24.5 \mathrm{~ms}, 20$-degree flip angle, $256 \times 168$ matrix, 230$\mathrm{mm}$ field of view, 5-mm-thick sections with 1-mm interslice gaps, and number of excitations 1 (acquisition time $=1: 31$ ). The parameters of multi-shot diffusion-weighted echo planar imaging were TR 3,500 ms, TE $94 \mathrm{~ms}, 128 \times 96$ matrix, 230-mm field of view, 5-mm-thick sections with 1-mm interslice gaps, number of excitations 2 , PAT factor 2 , b values of 0 , and $1,500 \mathrm{~s} / \mathrm{mm}^{2}$ (acquisition time $=0: 40$ ).

\section{Data Processing and Analysis}

Data were evaluated with commercially available 3D perfusion software (Syngo Volume Perfusion CT Neuro; Siemens, Forchheim, Germany). The signal-to-noise ratio was improved using spatiotemporal filtering for all data (adaptive 4D noise reduction). Vessels were automatically detected on the time maximum intensity projection images (fig. 1a). All voxels along the vasculature above a configured percentage of maximum enhancements were excluded from the calculation.

$\mathrm{CBF}, \mathrm{CBV}$, and MTT were determined with a deconvolution-based algorithm (fig. 1b-d) [28]. TTP was determined as the time lag indicated by the time attenuation curve (TAC) (fig. 1f R1-R3) between the injection of the contrast medium and the local bolus peak in the brain tissue. For example, TAC in the contralateral hemisphere, considered to indicate normal perfusion, showed a time interval of $17 \mathrm{~s}$ (fig. 1f R3), but R1 in the ischemic hemisphere showed a delayed time interval of $27 \mathrm{~s}$. According to the time lag, the color map was constructed as in figure 1e. However, in the most seriously ischemic part, TAC did not show any local bolus peak as in figure $1 \mathrm{f} \mathrm{R} 2$, which was interpreted as showing that the contrast medium did not sufficiently penetrate into the brain tissue because of extreme hypoperfusion. 


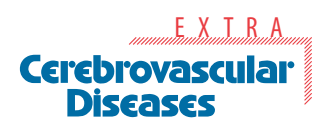

\begin{tabular}{l|l}
\hline \multicolumn{2}{l}{ Cerebrovasc Dis Extra 2013;3:14-25 } \\
\hline DOI: $\underline{10.1159 / 000346113}$ & $\begin{array}{l}\text { (c) 2013 S. Karger AG, Basel } \\
\text { www.karger.com/cee }\end{array}$ \\
\hline
\end{tabular}

Shinoyama et al.: Initial 'TTP Map-Defect' of Computed Tomography Perfusion as a Predictor of Hemorrhagic Transformation of Acute Ischemic Stroke

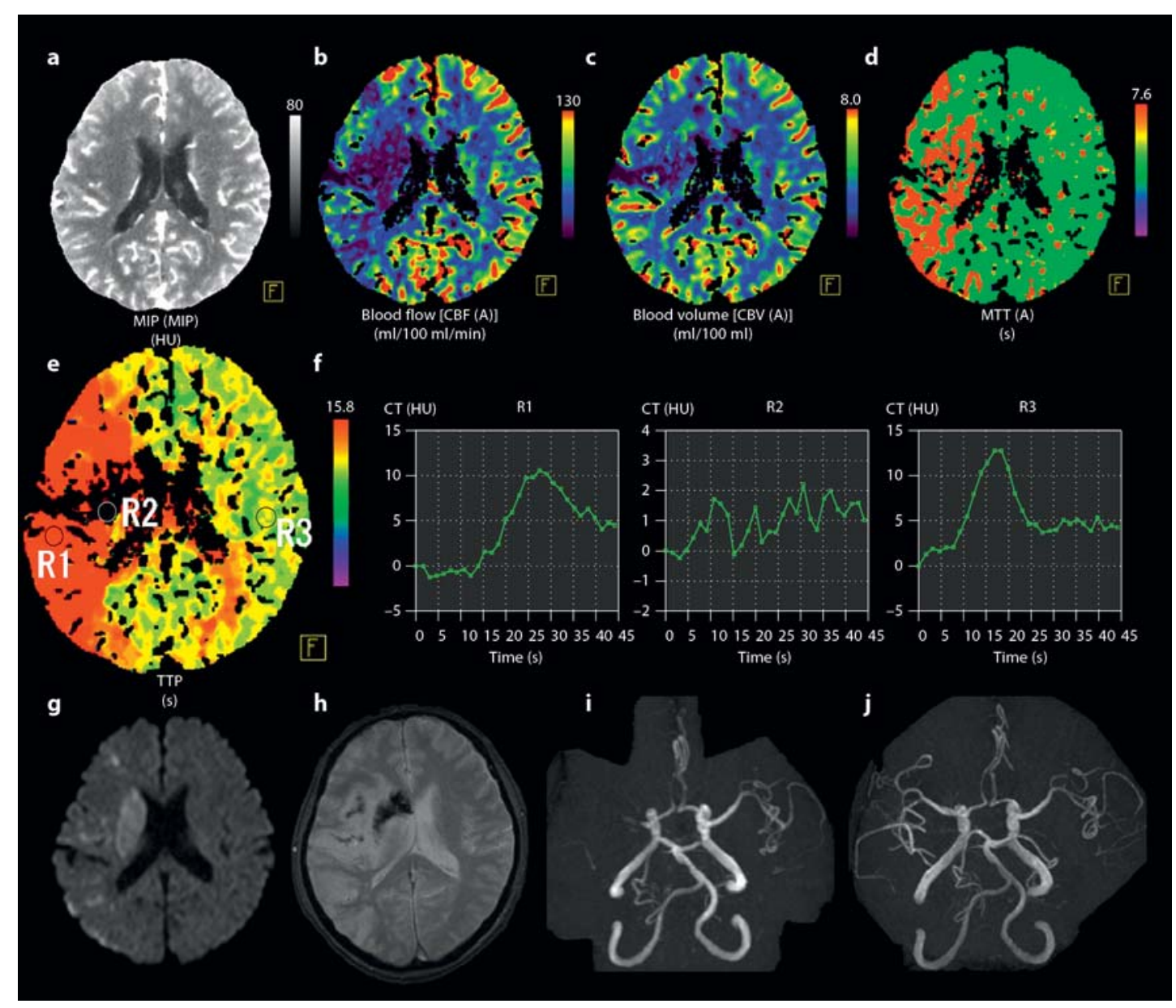

Fig. 1. A 70-year-old man with right middle cerebral artery (M1) occlusion who achieved spontaneous recanalization and developed $\mathrm{PH}$. a Maximum intensity projection (MIP) image showing no apparent enhanced lesion. b CBF map showing hypoperfusion. c CBV map showing low blood volume, and d MTT map showing delayed transit time in a large part of the right middle cerebral artery territory, but the area intended to develop HT was not visible in these images. e TTP map demonstrating the 'defect' area surrounded by areas with delayed time interval in the same region. $f$ Analysis of TAC showing a normal curve (R3), delayed curve (R1), and undetectable local bolus peak (R2). x-axis: time interval from injection, $y$ axis: Hounsfield unit (HU). g Initial diffusion-weighted image showing vague high intensity indicating acute cerebral infarction. $\mathbf{h}$ T2*-weighted imaging on day 10 demonstrating secondary HT in the same area as the initial 'TTP map-defect'. Initial MRA showing the right M1 occlusion (i) and follow-up MRA on day 10 showing complete recanalization (modified Mori scale grade 3) (j).

In this situation, we could not transfer areas with any delayed time interval onto the TTP map. Therefore, we defined these defect areas on the initial TTP map as 'TTP map-defect', which was clearly distinguishable from the surrounding area of moderate hypoperfusion (fig. 1e).

\section{Image Analysis}

The presence of HT was assessed on initial and follow-up T2*-weighted images using the standard MRI criteria for hemorrhage, and HT was also assessed on any unscheduled CT scans obtained at the occasion of any clinical deterioration. T2*-weighted imaging showed hemorrhagic infection (HI) as low-intensity signal within the ischemic area, within four cat- 
egories according to the European Cooperative Acute Stroke Study (ECASS) classification [2]: (1) HI1 (hemorrhagic infarction 1) defined as small petechiae along the margins of the infarct; (2) HI2 (hemorrhagic infarction 2) defined as confluent petechiae within the infarcted area but no space-occupying effect; (3) PH1 (parenchymal hemorrhage 1) defined as blood clots within $30 \%$ of the infarcted area with some slight space-occupying effect, and (4) PH2 (parenchymal hemorrhage 2) defined as blood clots in over $30 \%$ of the infarcted area with a substantial space-occupying effect (fig. 1h).

Symptomatic intracerebral hemorrhage $(\mathrm{sICH})$ was defined as clinical deterioration with an increase of more than four points in the National Institutes of Health Stroke Scale (NIHSS) score, if the hemorrhage was likely to be the cause of the clinical deterioration [1]. Recanalization of occluded major trunks was also assessed on follow-up MRA according to the modified Mori grade [29]: grade 0, no reperfusion; grade 1, movement of the thrombus not associated with any flow improvement; grade 2, partial (branch) recanalization in less than $50 \%$ of the branches in the occluded arterial territory, and grade 3 , nearly complete recanalization with reperfusion in more than $50 \%$ of the branches in the occluded arterial territory (fig. 1). In this study, grades 2 and 3 were defined as significant recanalization. All images were assessed by two reviewers, one expert neurosurgeon (M. Shinoyama) and one expert neuroradiologist (H. Ono) unaware of the clinical information except for the affected side.

\section{Review of Clinical Data}

Patients underwent clinical assessment with the NIHSS on admission and discharge, and with the modified Rankin Scale on discharge. We reviewed the patients' clinical data: baseline neurological deficits as assessed by the NIHSS score; history of hypertension, cardioembolic stroke risk factors such as atrial fibrillation, and diabetes; and current smoking or alcohol intake. In addition, laboratory data such as platelet count, prothrombin time-international normalized ratio (PT-INR), activated partial thromboplastin time (APTT), and serum glucose level were also assessed.

\section{Statistical Analysis}

Categorical data were analyzed with the two-tailed $\chi^{2}$ test. Continuous variables were analyzed with the unpaired Student $t$ test or, in case of abnormally distributed data, with the Mann-Whitney U test. Statistical analysis was performed with commercially available software (SPSS v17). A p value of less than 0.05 was considered to indicate a significant difference.

\section{Results}

\section{Patient Characteristics}

A total of 68 patients, 41 males and 27 females aged $72.9 \pm 11.7$ years (mean \pm SD) fulfilling the study criteria, were admitted to the stroke service of the Nakamura Memorial Hospital between January and September 2010. The overall median NIHSS score was 7 (range 0-30, mean 8.8).

Ten of the 68 patients (14.7\%) were treated with IV rt-PA $(0.6 \mathrm{mg} / \mathrm{kg})$ within $3 \mathrm{~h}$ of stroke onset according to the Japanese guidelines for rt-PA use. Twenty-eight patients (41\%) had been transferred within $3 \mathrm{~h}$ from stroke onset, but 18 were excluded from receiving IV rt-PA according to the Japanese guidelines.

The clinical characteristics of the patients with or without HT are shown in table 1. Secondary HT developed within 2 weeks of follow-up MRI (HT group) in 34 of the 68 patients (50\%), including $18 \mathrm{HI}$ patients (52.9\%: HI $\mathrm{n}=6,17.6 \%$; HI2 $\mathrm{n}=12,35.3 \%)$ and $16 \mathrm{PH}$ pa- 


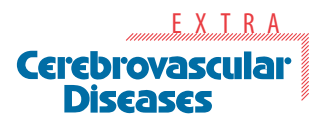

Table 1. Patient characteristics

\begin{tabular}{|c|c|c|c|}
\hline & Non-HT group $(\mathrm{n}=34)$ & HT group $(n=34)$ & $\mathrm{p}$ \\
\hline Mean age $\pm S D$, years & $72.3 \pm 12.6$ & $73.7 \pm 11.2$ & 0.6611 \\
\hline Age $>65$ years, $\mathrm{n}(\%)$ & $25(73.5)$ & $27(79.4)$ & 0.5675 \\
\hline Male gender, n (\%) & $24(70.6)$ & $17(50)$ & 0.0828 \\
\hline \multicolumn{4}{|l|}{ Type of infarction, $\mathrm{n}(\%)$} \\
\hline Cardioembolic & $14(41.2)$ & $29(85.3)$ & 0.0002 \\
\hline Atherothrombotic & $18(52.9)$ & $5(14.7)$ & 0.0003 \\
\hline Undetermined & $2(5.9)$ & $0(0)$ & 0.1512 \\
\hline \multicolumn{4}{|l|}{ Site of thrombus, $\mathrm{n}(\%)$} \\
\hline ICA & $9(26.5)$ & $6(17.6)$ & 0.3803 \\
\hline ACA A1 & $3(8.8)$ & $1(2.9)$ & 0.3026 \\
\hline MCA M1 & $9(26.5)$ & $16(47.1)$ & 0.0783 \\
\hline MCA M2 & $8(23.5)$ & $8(23.5)$ & 1 \\
\hline PCA P2 & $2(5.9)$ & $3(8.8)$ & 0.6422 \\
\hline VA-BA system & $3(8.8)$ & $0(0)$ & 0.0765 \\
\hline \multicolumn{4}{|l|}{ Medical history, n (\%) } \\
\hline Diabetes mellitus & $8(23.5)$ & $11(32.4)$ & 0.4175 \\
\hline Hypertension & $23(67.7)$ & $21(61.8)$ & 0.6118 \\
\hline Hypercholesterolemia & $8(23.5)$ & $10(29.4)$ & 0.5825 \\
\hline Atrial fibrillation & $7(20.6)$ & $22(64.7)$ & 0.0001 \\
\hline Stroke & $6(17.7)$ & $5(14.7)$ & 0.7419 \\
\hline Current smoking, n (\%) & $9(26.5)$ & $8(23.5)$ & 0.7794 \\
\hline Current alcohol use, n (\%) & $5(14.7)$ & $6(17.7)$ & 0.7419 \\
\hline \multicolumn{4}{|l|}{ Pre-stroke drug, n (\%) } \\
\hline Antiplatelet & $5(14.7)$ & $7(20.6)$ & 0.3549 \\
\hline Anticoagulant & $3(8.8)$ & $7(20.6)$ & 0.0996 \\
\hline \multicolumn{4}{|l|}{ Radiological findings, $\mathrm{n}(\%)$} \\
\hline TTP map-defect & $6(17.6)$ & $34(100)$ & 0.0001 \\
\hline Recanalization & $8(23.6)$ & $30(88.2)$ & 0.0001 \\
\hline \multicolumn{4}{|l|}{ Medication } \\
\hline IV rt-PA & $5(14.7)$ & $5(14.7)$ & 1 \\
\hline \multicolumn{4}{|l|}{ On admission } \\
\hline Mean NIHSS score \pm SD & $5.1 \pm 5.6$ & $12.5 \pm 7.2$ & 0.0001 \\
\hline NIHSS score $>6, \mathrm{n}(\%)$ & $11(32.4)$ & $29(85.3)$ & 0.0001 \\
\hline \multicolumn{4}{|l|}{ On discharge } \\
\hline Mean NIHSS score \pm SD & $2.4 \pm 3.9$ & $8.0 \pm 7.0$ & 0.0001 \\
\hline NIHSS score $>6, \mathrm{n}(\%)$ & $6(17.6)$ & $18(52.9)$ & 0.0023 \\
\hline mRS score $0-1, \mathrm{n}(\%)$ & $20(58.8)$ & $7(20.6)$ & 0.0013 \\
\hline mRS score $0-2, \mathrm{n}(\%)$ & $23(67.7)$ & $11(32.5)$ & 0.0036 \\
\hline
\end{tabular}

ICA = Internal carotid artery; $\mathrm{ACA}=$ anterior cerebral artery; $\mathrm{MCA}=$ middle cerebral artery; $\mathrm{PCA}=$ posterior cerebral artery; VA-BA = vertebrobasilar; $\mathrm{mRS}=$ modified Rankin Scale.

tients (47.1\%: PH1 n = 7, 20.6\%; PH2 n = 9, 26.5\%), but not in the other 34 patients (non-HT group). There was no significant difference in the incidence of HT between patients treated and not treated with IV rt-PA. Four of the $34 \mathrm{HT}$ patients $(11.8 \%)$ had sICH, all of ECASS category $\mathrm{PH} 2$. One of these 4 patients with sICH underwent IV rt-PA. Fifteen of the 34 patients (44.1\%) with HT revealed a hemorrhagic lesion on day 2 on the first follow-up MRI, and the other 19 patients on the second follow-up MRI.

Significantly more patients had a cardioembolic etiology in the HT group, whereas significantly fewer patients had an atherothrombotic etiology. Analysis of the patients' medical history showed that only atrial fibrillation was significantly more frequent in the 

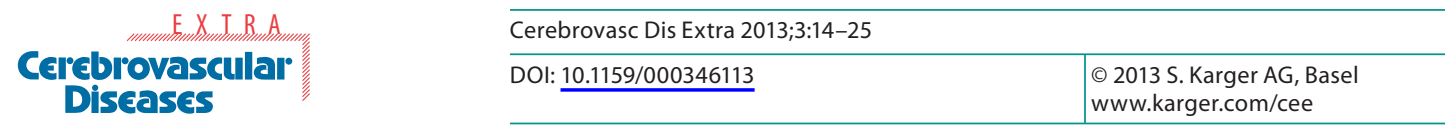

Shinoyama et al.: Initial 'TTP Map-Defect' of Computed Tomography Perfusion as a Predictor of Hemorrhagic Transformation of Acute Ischemic Stroke

Table 2. Patient characteristics

\begin{tabular}{|c|c|c|c|}
\hline & Non-PH2 group $(n=59)$ & PH2 group $(n=9)$ & $\mathrm{p}$ \\
\hline Mean age \pm SD, years & $72.9 \pm 12.0$ & $73.6 \pm 9.2$ & 0.8511 \\
\hline Age $>65$ years, $\mathrm{n}(\%)$ & $45(76.3)$ & $7(77.8)$ & 0.9209 \\
\hline Male gender, $\mathrm{n}(\%)$ & $36(61.0)$ & $5(55.6)$ & 0.7551 \\
\hline \multicolumn{4}{|l|}{ Type of infarction, $\mathrm{n}(\%)$} \\
\hline Cardioembolic & $34(57.6)$ & $9(100)$ & 0.0141 \\
\hline Atherothrombotic & $23(39.0)$ & $0(0)$ & 0.0213 \\
\hline Undetermined & $2(3.4)$ & $0(0)$ & 0.5750 \\
\hline \multicolumn{4}{|l|}{ Site of thrombus, $\mathrm{n}(\%)$} \\
\hline ICA & $14(23.7)$ & $1(11.1)$ & 0.3951 \\
\hline ACA A1 & $4(6.8)$ & $0(0)$ & 0.4207 \\
\hline MCA M1 & $21(35.6)$ & $4(44.4)$ & 0.6080 \\
\hline MCA M2 & $14(23.7)$ & $2(22.2)$ & 0.4250 \\
\hline PCA P2 & $3(5.1)$ & $2(22.2)$ & 0.0665 \\
\hline VA-BA system & $3(5.1)$ & $0(0)$ & 0.4890 \\
\hline \multicolumn{4}{|l|}{ Medical history, n (\%) } \\
\hline Diabetes mellitus & $14(23.7)$ & $1(11.1)$ & 0.3951 \\
\hline Hypertension & $41(69.5)$ & $4(44.4)$ & 0.1390 \\
\hline Hypercholesterolemia & $15(25.4)$ & $3(33.3)$ & 0.6164 \\
\hline Atrial fibrillation & $26(44.1)$ & $4(44.4)$ & 0.9831 \\
\hline Stroke & $11(18.6)$ & $0(0)$ & 0.1571 \\
\hline Current smoking, n (\%) & $15(25.4)$ & $2(22.2)$ & 0.8363 \\
\hline Current alcohol use, $\mathrm{n}(\%)$ & $9(15.3)$ & $2(22.2)$ & 0.5970 \\
\hline \multicolumn{4}{|l|}{ Pre-stroke drug, n (\%) } \\
\hline Antiplatelet & $12(20.3)$ & $3(33.3)$ & 0.3812 \\
\hline Anticoagulant & $10(16.9)$ & $1(11.1)$ & 0.6577 \\
\hline \multicolumn{4}{|l|}{ Radiological findings, $\mathrm{n}(\%)$} \\
\hline TTP map-defect & $31(52.5)$ & $9(100)$ & 0.0070 \\
\hline Recanalization & $29(49.2)$ & $9(100)$ & 0.0042 \\
\hline \multicolumn{4}{|l|}{ Medication } \\
\hline IV rt-PA & $7(11.9)$ & $3(33.3)$ & 0.0903 \\
\hline \multicolumn{4}{|l|}{ On admission } \\
\hline Mean NIHSS score \pm SD & $8.6 \pm 7.5$ & $10.2 \pm 6.9$ & 0.5302 \\
\hline NIHSS score $>6, \mathrm{n}(\%)$ & $33(55.9)$ & $7(77.8)$ & 0.2148 \\
\hline \multicolumn{4}{|l|}{ On discharge } \\
\hline Mean NIHSS score \pm SD & $4.9 \pm 6.3$ & $6.2 \pm 6.0$ & 0.5489 \\
\hline NIHSS score $>6, \mathrm{n}(\overline{\%})$ & $19(32.2)$ & $5(55.6)$ & 0.1721 \\
\hline mRS score $0-1, \mathrm{n}(\%)$ & $24(40.7)$ & $2(22.2)$ & 0.2886 \\
\hline mRS score $0-2, \mathrm{n}(\%)$ & $30(50.8)$ & $4(44.4)$ & 0.7205 \\
\hline
\end{tabular}

$\mathrm{ICA}=$ Internal carotid artery; $\mathrm{ACA}=$ anterior cerebral artery; $\mathrm{MCA}=$ middle cerebral artery; $\mathrm{PCA}=$ posterior cerebral artery; VA-BA = vertebrobasilar; $\mathrm{mRS}=$ modified Rankin Scale.

HT group. Neurological findings and clinical outcomes showed significant differences between the HT and non-HT groups. The mean NIHSS score of the HT patients was significantly higher than that of the non-HT patients. Severe deficits (NIHSS score $>6$ ) on admission were also significantly associated with HT. Both the NIHSS and the modified Rankin Scale scores were significantly worse in the HT group at discharge. Administration of pre-stroke antiplatelet and anticoagulant agents tended to be more common in the HT group, but not significantly. Atrial fibrillation was significantly more common in the HT group, but only 7 patients $(20.6 \%)$ had undergone anticoagulant administration before stroke onset. 


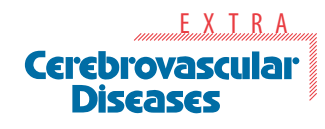

Table 3. Recanalization of patients with 'TTP map-defect'

\begin{tabular}{lccc}
\hline & \multicolumn{2}{l}{ Recanalization } & $\mathrm{p}$ \\
\cline { 2 - 3 } & yes & no & \\
\hline Non-HT group vs. HT group & $0(0)$ & $6(15.0)$ & $<0.0001$ \\
$\quad$ Non-HT & $30(75.0)$ & $4(10.0)$ & \\
$\quad$ HT & $21(52.5)$ & $10(25.0)$ & \\
Non-PH2 group vs. PH2 group & $9(22.5)$ & $0(0)$ & \\
$\quad$ Non-PH2 & & & \\
PH2 & & \\
\hline
\end{tabular}

The mean PT-INR on admission showed no differences between the groups (non-HT group vs. HT group: $1.15 \pm 0.24$ vs. $1.16 \pm 0.24$, mean $\pm S D, p=0.939$ ). In addition, laboratory examination on admission showed no significant differences in serum glucose level (non-HT group vs. HT group: $134.3 \pm 52.5$ vs. $125.2 \pm 38.2 \mathrm{mg} / \mathrm{dl}, \mathrm{p}=0.416$ ) and other coagulation parameters such as APTT (non-HT group vs. HT group: $29.9 \pm 3.7$ vs. $31.1 \pm$ $5.8 \mathrm{~s}, \mathrm{p}=0.327$ ) and platelet count (non-HT group vs. HT group: $16.8 \pm 4.6$ vs. $18.5 \pm 5.2$ $\times 10^{4} / \mu \mathrm{l}, \mathrm{p}=0.146$ ). No differences were found in age, sex, location of occluded site, history of diabetes, hypertension, hypercholesterolemia, and stroke, as well as current smoking or alcohol intake.

The clinical characteristics of the patients with or without PH2 are shown in table 2. Significantly more patients had a cardioembolic etiology in the PH2 group, whereas significantly fewer patients had an atherothrombotic etiology. There were no significant differences in the underlying medical histories and patients' clinical severity in contrast to the results of the HT versus non-HT group analysis. Mean PT-INR of the non-PH2 group was significantly higher than that of the $\mathrm{PH} 2$ group $(1.16 \pm 0.25$ vs. $1.08 \pm 0.06, \mathrm{p}=0.029)$. Moreover, serum glucose level of the non-PH2 group was lower than that of the $\mathrm{PH} 2$ group $(127.1 \pm 47.8$ vs. $147.0 \pm 17.5 \mathrm{mg} / \mathrm{dl}, \mathrm{p}=0.032)$. In contrast, coagulation parameters such as APTT (non-PH2 group vs. PH2 group: $30.8 \pm 5.0$ vs. $28.2 \pm 2.8 \mathrm{~s}, \mathrm{p}=0.062$ ) and platelet count (non-PH2 group vs. PH2 group: $18.0 \pm 4.7$ vs. $15.4 \pm 5.6 \times 10^{4} / \mu \mathrm{l}, \mathrm{p}=0.238$ ) showed no significant differences.

\section{Imaging Findings}

The radiological findings are also shown in tables 1 and 2 . Forty patients revealed 'TTP map-defect' on the initial CTP map, and 34 of these 40 patients (85\%) developed HT (table 1). Of these 40 patients, 9 patients (22.5\%) showed PH2 (table 2). In the HT group, 30 of 34 patients (88.2\%) experienced delayed recanalization of the occluded vessels on follow-up MRA until 1 week after onset in contrast to only 8 of 34 patients (23.6\%) in the non-HT group (table 1). In the $\mathrm{PH} 2$ group, all patients showed delayed recanalization.

Table 3 shows the association between recanalization and following hemorrhagic outcome (HT or $\mathrm{PH} 2$ ) of 40 patients with initial 'TTP map-defect'. In these positive patients, recanalization caused significant HT or PH2. During the analysis for HT, 30 patients (75\%) with recanalization developed HT. In the other 10 patients without recanalization, 6 patients (15\%) did not develop HT and 4 patients (10\%) showed HI, but no PH (HI1 n = 1, HI2 $\mathrm{n}=3$ ). Patients with both 'TTP map-defect' and delayed recanalization subsequently developed HT without exception, and the areas of HT were consistent with the 'TTP mapdefect' on the initial CTP map (fig. 1). Nine patients (22.5\%) with recanalization developed $\mathrm{PH} 2$ (table 3). There were no patients with 'TTP map-defect' and without delayed recana- 


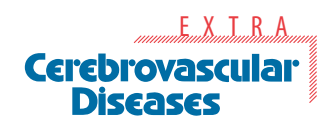

Shinoyama et al.: Initial 'TTP Map-Defect' of Computed Tomography Perfusion as a

Predictor of Hemorrhagic Transformation of Acute Ischemic Stroke

lization, resulting in PH2. All 28 patients without 'TTP map-defect' did not develop HT, including 8 patients (28.6\%) with delayed recanalization (data not shown).

\section{Neurological Findings and Prognosis}

There were significant differences in patients' neurological severity on admission or discharge between the positive and negative 'TTP map-defect' groups. The initial mean NIHSS score of the patients with 'TTP map-defect' was significantly higher than that of the patients without defect (positive vs. negative 'TTP map-defect' group: $12.2 \pm 7.2$ vs. $4.0 \pm 4.6$, p < 0.001 ). Severe deficits (NIHSS score $>6$ ) on admission were also significantly associated with 'TTP map-defect' (positive vs. negative group: 32 vs. $6, \mathrm{p}<0.001$ ). The functional outcomes of the 'TTP map-defect'-positive group were severer than those of the negative group (positive vs. negative group: modified Rankin Scale 0-1, 8 vs. 18, p < 0.001; modified Rankin Scale $0-2,12$ vs. $22, \mathrm{p}<0.001)$.

\section{Discussion}

This is the first study demonstrating that initial TTP mapping on admission could identify patients at risk of HT following acute ischemic stroke. All patients with HT had shown 'TTP map-defect' on the initial CTP map, whereas patients without 'TTP map-defect' did not develop any HT irrespective of vessel recanalization. These findings suggest that stroke without 'TTP map-defect' might involve the non-stagnant vasculature, which could be due to the rich collateral flow, with certain tolerance against reperfusion even if delayed. The present findings indicate the diagnostic efficacy of TTP mapping for the estimation of the HT risk after acute ischemic stroke.

Several radiological parameters have been evaluated to predict HT [7], based on various aspects such as CBF, tissue perfusion, blood-brain barrier, brain edema, and others. Singlephoton emission CT indicating low CBF has been reported to predict the risk of HT [30-32]. A significant relationship was first identified between qualitative CBF analyzed by ${ }^{99 \mathrm{~m} T \mathrm{~T}-}$ labeled hexamethylpropyleneamine oxime single-photon emission CT and subsequent HT [30]. Residual CBF of less than 35\% in the ipsilateral cerebellum also showed high risk of HT using the same modality in a series of patients with intra-arterial thrombolysis [31, 32]. Furthermore, in studies using perfusion imaging, the volume of a low CBV lesion may be closely correlated with HT risk [14]. Prolonged relative MTT was also reported as independent predictor of secondary HT [15]. Our results were encouraged by these previous studies showing correlations between local hypoperfusion and high HT risk.

The present study showed that 'TTP map-defect' could clearly classify patients into a high-HT risk group (85\% HT risk, 22.5\% PH2 risk) and a low-HT risk group (0\% HT risk). The sensitivity of 'TTP map-defect' for the occurrence of HT or PH2 was 100\%, and the specificity was 82.4 and $47.5 \%$, respectively. These findings are consistent with previous animal studies, which showed that extreme hypoperfusion resulted in damage to the bloodbrain barrier integrity, especially endothelial tight junction disruption, and the development of HT $[33,34]$.

Findings of TTP mapping may alter the indication of thrombolytic therapy. The therapeutic time window of IV rt-PA is supposed to be 3-4.5 h from the onset [35], but it might depend on both the recanalization rate of reperfusion treatment and the local ischemic threshold. For instance, a much wider time window has been proposed in patients with arterial thrombolysis [36] or posterior circular lesion [37]. In this context, we emphasize that TTP mapping could differentiate low-HT risk patients from high-HT risk patients within the therapeutic time window frame to achieve safe reperfusion treatment. Moreover, TTP map- 


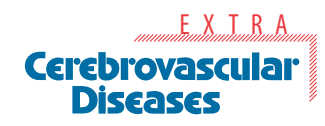

ping may identify patients with low HT risk, even if they have a delayed arrival time beyond $4.5 \mathrm{~h}$.

On the other hand, findings of TTP mapping may also alter post-thrombolytic management. Previous reports have shown that elevation or variability of blood pressure correlated to HT development in patients with or without IV rt-PA [38-40]. We propose that the blood pressure level of patients with 'TTP map-defect' should be controlled strictly and be more stable and lower than that of patients without 'TTP map-defect'. Although knowledge on HT risk could be useful, further prospective and large-size examinations are needed for confirming the efficacy of TTP mapping in the management of pre- or post-thrombolytic therapy.

Perfusion imaging, such as CTP and MRP, is superior to other modalities in terms of fewer limitations of time, place, drug supply, or duration of examination. In particular, CTP by multi-detector row CT can provide efficient spatial resolution of whole-brain imaging. For instance, even HT risk caused by small lesions associated with distal branch occlusion, such as downstream of M2, could be clearly detected by CTP, but not by conventional CT or MRI [7]. In the future, the presence or absence of a 'TTP map-defect' should be confirmed using MRP to avoid the exposure to radiation required by CTP. Additionally, MRP can be obtained with various parameters including CBV, permeability, and apparent diffusion coefficient value at the same time and in the same plane, thus allowing a multi-imaging assessment for reperfusion treatment.

The present study has several limitations. One major limitation is the small sample size and retrospective nature, with potential bias. Previously identified clinical predictors were not correlated with HT in this series, which is probably attributable to the small sample size. Moreover, we could not assess the association between symptomatic and asymptomatic ICH because of the small number of sICH cases $(n=4)$. Further, the small sample size also restricted analysis of the variables including 'TTP-map defect' using multivariate analysis. While our results are suggestive, a prospective trial with more patients is required to reinforce this preliminary finding of 'TTP map-defect' as a predictor of HT. The second limitation is the assessment procedure. We did not evaluate patient variables such as imaging, neurology, and outcome in a blind procedure. The third limitation is that our procedure could not be applied to patients with renal failure because of the toxicity of the contrast medium.

In conclusion, our study shows that the initial 'TTP-map defect' of CTP is able to predict HT following acute ischemic stroke. TTP mapping can differentiate low-HT risk patients within the therapeutic time window frame or with unknown onset from patients with high HT risk and allows safe administration of reperfusion treatment.

\section{References}

1 Moulin T, Crépin-Leblond T, Chopard JL, Bogousslavsky J: Hemorrhagic infarcts. Eur Neurol 1994; 34:64-77.

-2 Hacke W, Kaste M, Fieschi C, Toni D, Lesaffre E, von Kummer R, Boysen G, Bluhmki E, Höxter G, Mahagne MH, Hennerici M, ECASS Study Group: Intravenous thrombolysis with recombinant tissue plasminogen activator for acute hemispheric stroke. The European Cooperative Acute Stroke Study (ECASS). JAMA 1995;274:1017-1025.

-3 The National Institute of Neurological Disorders and Stroke rt-PA Stroke Study Group: Tissue plasminogen activator for acute ischemic stroke. N Engl J Med 1995;333:1581-1587.

-4 Donnan GA, Davis SM, Parsons MW, Ma H, Dewey HM, Howells DW: How to make better use of thrombolytic therapy in acute ischemic stroke. Nat Rev Neurol 2011;7:400-409. 
Shinoyama et al.: Initial 'TTP Map-Defect' of Computed Tomography Perfusion as a

Predictor of Hemorrhagic Transformation of Acute Ischemic Stroke

-5 Adeoye O, Hornung R, Khatri P, Kleindorfer D: Recombinant tissue-type plasminogen activator use for ischemic stroke in the United States: a doubling of treatment rates over the course of 5 years. Stroke 2011;42:1952-1955.

-6 Del Zoppo GJ, Saver JL, Jauch EC, Adams HP Jr, American Heart Association Stroke Council: Expansion of the time window for treatment of acute ischemic stroke with intravenous tissue plasminogen activator: a science advisory from the American Heart Association/American Stroke Association. Stroke 2009;40:2945-2948.

7 Derex L, Nighoghossian N: Intracerebral haemorrhage after thrombolysis for acute ischaemic stroke: an update. J Neurol Neurosurg Psychiatry 2008;79:1093-1099.

8 Lansberg MG, Thijs VN, Bammer R, Kemp S, Wijman CA, Marks MP, Albers GW, DEFUSE Investigators: Risk factors of symptomatic intracerebral hemorrhage after tPA therapy for acute stroke. Stroke 2007;38:2275-2278.

-9 Lansberg MG, Albers GW, Wijman CA: Symptomatic intracerebral hemorrhage following thrombolytic therapy for acute ischemic stroke: a review of the risk factors. Cerebrovasc Dis 2007;24:1-10.

-10 Larrue V, von Kummer RR, Müller A, Bluhmki E: Risk factors for severe hemorrhagic transformation in ischemic stroke patients treated with recombinant tissue plasminogen activator: a secondary analysis of the European-Australasian Acute Stroke Study (ECASS II). Stroke 2001;32:438-441.

- 11 Kablau M, Kreisel SH, Sauer T, Binder J, Szabo K, Hennerici MG, Kern R: Predictors and early outcome of hemorrhagic transformation after acute ischemic stroke. Cerebrovasc Dis 2011;32:334-341.

-12 Lansberg MG, Albers GW, Wijman CA: Symptomatic intracerebral hemorrhage following thrombolytic therapy for acute ischemic stroke: a review of the risk factors. Cerebrovasc Dis 2007;24:1-10.

-13 Wintermark M, Reichhart M, Thiran JP, Maeder P, Chalaron M, Schnyder P, Bogousslavsky J, Meuli R: Prognostic accuracy of cerebral blood flow measurement by perfusion computed tomography, at the time of emergency room admission, in acute stroke patients. Ann Neurol 2002;51:417-432.

- 14 Sasaki M, Kudo K, Honjo K, Hu JQ, Wang HB, Shintaku K: Prediction of infarct volume and neurologic outcome by using automated multiparametric perfusion-weighted magnetic resonance imaging in a primate model of permanent middle cerebral artery occlusion. J Cereb Blood Flow Metab 2011; 31:448-456.

-15 Lin K, Do KG, Ong P, Shapiro M, Babb JS, Siller KA, Pramanik BK: Perfusion CT improves diagnostic accuracy for hyperacute ischemic stroke in the 3-hour window: study of 100 patients with diffusion MRI confirmation. Cerebrovasc Dis 2009;28:72-79.

-16 Agarwal S, Jones PS, Alawneh JA, Antoun NM, Barry PJ, Carrera E, Cotter PE, O’Brien EW, Salih I, Scoffings DJ, Baron JC, Warburton EA: Does perfusion computed tomography facilitate clinical decision making for thrombolysis in unselected acute patients with suspected ischaemic stroke? Cerebrovasc Dis 2011;32:227-233.

-17 Aviv RI, d'Esterre CD, Murphy BD, Hopyan JJ, Buck B, Mallia G, Li V, Zhang L, Symons SP, Lee TY: Hemorrhagic transformation of ischemic stroke: prediction with CT perfusion. Radiology 2009;250: 867-877.

18 Campbell BC, Christensen S, Butcher KS, Gordon I, Parsons MW, Desmond PM, Barber PA, Levi CR, Bladin CF, De Silva DA, Donnan GA, Davis SM, EPITHET Investigators: Regional very low cerebral blood volume predicts hemorrhagic transformation better than diffusion-weighted imaging volume and thresholded apparent diffusion coefficient in acute ischemic stroke. Stroke 2010;41:8288.

-19 Souza LC, Payabvash S, Wang Y, Kamalian S, Schaefer P, Gonzalez RG, Furie KL, Lev MH: Admission CT perfusion is an independent predictor of hemorrhagic transformation in acute stroke with similar accuracy to DWI. Cerebrovasc Dis 2012;33:8-15.

-20 Sobesky J, Zaro Weber O, Lehnhardt FG, Hesselmann V, Thiel A, Dohmen C, Jacobs A, Neveling M, Heiss WD: Which time-to-peak threshold best identifies penumbral flow? A comparison of perfusion-weighted magnetic resonance imaging and positron emission tomography in acute ischemic stroke. Stroke 2004;35:2843-2847.

-21 Albers GW, Thijs VN, Wechsler L, Kemp S, Schlaug G, Skalabrin E, Bammer R, Kakuda W, Lansberg MG, Shuaib A, Coplin W, Hamilton S, Moseley M, Marks MP, DEFUSE Investigators: Magnetic resonance imaging profiles predict clinical response to early reperfusion: the diffusion and perfusion imaging evaluation for understanding stroke evolution (DEFUSE) study. Ann Neurol 2006;60:508517 . 
Shinoyama et al.: Initial 'TTP Map-Defect' of Computed Tomography
Predictor of Hemorrhagic Transformation of Acute Ischemic Stroke

-22 Olivot JM, Mlynash M, Thijs VN, Kemp S, Lansberg MG, Wechsler L, Bammer R, Marks MP, Albers GW: Optimal Tmax threshold for predicting penumbral tissue in acute stroke. Stroke 2009;40:469475.

-23 Reichenbach JR, Röther J, Jonetz-Mentzel L, Herzau M, Fiala A, Weiller C, Kaiser WA: Acute stroke evaluated by time-to-peak mapping during initial and early follow-up perfusion CT studies. AJNR Am J Neuroradiol 1999;20:1842-1850.

-24 Perez-Arjona EA, DelProposto Z, Sehgal V, Fessler RD: New techniques in cerebral imaging. Neurol Res 2002;24(suppl 1):S17-S26.

-25 Berger C, Fiorelli M, Steiner T, Schäbitz WR, Bozzao L, Bluhmki E, Hacke W, von Kummer R: Hemorrhagic transformation of ischemic brain tissue: asymptomatic or symptomatic? Stroke 2001;32: 1330-1335.

26 The Joint Committee on Guidelines for the Management of Stroke: Japanese Guidelines for the Management of Stroke 2009. http://www.jsts.gr.jp/jss08.html.

-27 Yamaguchi T, Mori E, Minematsu K, Nakagawara J, Hashi K, Saito I, Shinohara Y, Japan Alteplase Clinical Trial (J-ACT) Group: Alteplase at $0.6 \mathrm{mg} / \mathrm{kg}$ for acute ischemic stroke within $3 \mathrm{~h}$ of onset: Japan Alteplase Clinical Trial (J-ACT). Stroke 2006;37:1810-1815.

-28 Eastwood JD, Provenzale JM, Hurwitz LM, Lee TY: Practical injection-rate CT perfusion imaging: deconvolution-derived hemodynamics in a case of stroke. Neuroradiology 2001;43:223-226.

-29 Mori E, Minematsu K, Nakagawara J, Yamaguchi T, Sasaki M, Hirano T, Japan Alteplase Clinical Trial II Group: Effects of $0.6 \mathrm{mg} / \mathrm{kg}$ intravenous alteplase on vascular and clinical outcomes in middle cerebral artery occlusion: Japan Alteplase Clinical Trial II (J-ACT II). Stroke 2010;41:461-465.

-30 Alexandrov AV, Black SE, Ehrlich LE, Caldwell CB, Norris JW: Predictors of hemorrhagic transformation occurring spontaneously and on anticoagulants in patients with acute ischemic stroke. Stroke 1997;28:1198-1202.

- 31 Ueda T, Hatakeyama T, Kumon Y, Sakaki S, Uraoka T: Evaluation of risk of hemorrhagic transformation in local intra-arterial thrombolysis in acute ischemic stroke by initial SPECT. Stroke 1994;25: 298-303.

- 32 Ueda T, Sakaki S, Yuh WT, Nochide I, Ohta S: Outcome in acute stroke with successful intra-arterial thrombolysis and predictive value of initial single-photon emission-computed tomography. J Cereb Blood Flow Metab 1999;19:99-108.

-33 Dijkhuizen RM, Asahi M, Wu O, Rosen BR, Lo EH: Delayed rt-PA treatment in a rat embolic stroke model: diagnosis and prognosis of ischemic injury and hemorrhagic transformation with magnetic resonance imaging. J Cereb Blood Flow Metab 2001;21:964-971.

-34 Neumann-Haefelin C, Brinker G, Uhlenküken U, Pillekamp F, Hossmann KA, Hoehn M: Prediction of hemorrhagic transformation after thrombolytic therapy of clot embolism: an MRI investigation in rat brain. Stroke 2002;33:1392-1398.

-35 Hacke W, Kaste M, Bluhmki E, Brozman M, Dávalos A, Guidetti D, Larrue V, Lees KR, Medeghri Z, Machnig T, Schneider D, von Kummer R, Wahlgren N, Toni D, ECASS Investigators: Thrombolysis with alteplase 3 to $4.5 \mathrm{~h}$ after acute ischemic stroke. N Engl J Med 2008;359:1317-1329.

-36 Furlan M, Marchal G, Viader F, Derlon JM, Baron JC: Spontaneous neurological recovery after stroke and the fate of the ischemic penumbra. Ann Neurol 1996;40:216-226.

-37 Hacke W, Zeumer H, Ferbert A, Brückmann H, del Zoppo GJ: Intra-arterial thrombolytic therapy improves outcome in patients with acute vertebrobasilar occlusive disease. Stroke 1988;19:12161222.

-38 Yong M, Kaste M: Association of characteristics of blood pressure profiles and stroke outcomes in the ECASS-II trial. Stroke 2008;39:366-372.

-39 Butcher K, Christensen S, Parsons M, De Silva DA, Ebinger M, Levi C, Jeerakathil T, Campbell BC, Barber PA, Bladin C, Fink J, Tress B, Donnan GA, Davis SM, EPITHET Investigators: Postthrombolysis blood pressure elevation is associated with hemorrhagic transformation. Stroke 2010;41:7277.

-40 Ko Y, Park JH, Yang MH, Ko SB, Han MK, Oh CW, Lee J, Lee J, Bae HJ: The significance of blood pressure variability for the development of hemorrhagic transformation in acute ischemic stroke. Stroke 2010;41:2512-2518. 